\title{
Battlefield Acupuncture in the Veterans Health Administration: Effectiveness in Individual and Group Settings for Pain and Pain Comorbidities
}

\author{
Daniel Glenn Federman, MD, ${ }^{1,2}$ Steven B. Zeliadt, PhD, MPH, ${ }^{3,4}$ Eva R. Thomas, MPH, \\ Gennaro F. Carbone Jr., BA, and Stephanie L. Taylor, $\mathrm{PhD}^{5,6}$
}

\begin{abstract}
Objective: The Department of Veterans Affairs trained primary-care providers to deliver Battlefield Acupuncture (BFA), a subset of auricular acupuncture, to patients. However, little is known about BFA effectiveness in group or individual sessions or repeated administrations versus singular use. The aim of this study was to examine the use and effectiveness of BFA for back pain and four pain-comorbid conditions in group and individual sessions at a large Veterans Affairs (VA) medical center.

Materials and Methods: This cross-sectional study was conducted at the West Haven VA Medical Center, in West Haven CT. Between October 2016 and December 2017, 284 veterans with pain received BFA. The BFA was administered in group clinics or in individual encounters. The Defense and Veterans Pain Rating Scale was used to assess self-reported pain immediately before and after each BFA administration.

Results: Over the study period, an average of 57 (range: 50-66) new patients per month received BFA. Of 753 total patient encounters, an immediate decrease in self-reported pain occurred in $616(82.0 \%)$ patients, no change occurred in $73(9.7 \%)$ patients, and an increase occurred in $62(8.3 \%)$ patients. Decreases in pain were common in the group and individual settings, even in patients with originally high pain scores, and the effectiveness remained with repeated uses.

Conclusions: BFA can be effective for immediate relief of pain-whether the BFA is administered in a group or individual setting - for the overwhelming majority of veterans and, as such, holds promise as a nonpharmacologic pain-management intervention.
\end{abstract}

Keywords: acupuncture, pain, Battlefield Acupuncture, veterans

\section{INTRODUCTION}

$\mathbf{P}$ AIN Is a Very COMMON patient complaint, both in veteran and nonveteran populations. Among the 5.7 million unique patients seen annually within the Department of Veterans Affairs (DVA), more than half of these patients experience chronic pain. ${ }^{1}$ Pharmacologic therapy, especially the use of opioids, is fraught with potential problems, and opioids have been linked to addiction, diversion, and death. Despite this knowledge, the mortality rate from prescription

\footnotetext{
${ }^{1}$ Department of Medicine, VA Connecticut Healthcare System, West Haven, CT.

${ }^{2}$ Yale University School of Medicine, New Haven, CT.

${ }^{3}$ Center of Innovation for Veteran-Centered and Value-Driven Care, VA Puget Sound HealthCare System, Seattle, WA.

${ }^{4}$ Department of Health Services, School of Public Health, University of Washington, Seattle, WA.

${ }^{5}$ VA Greater Los Angeles Healthcare System, West Los Angeles, CA.

${ }^{6}$ Department of Health Policy and Management, University of California-Los Angeles, Los Angeles, CA.

The views expressed in this article are those of the authors and do not necessarily represent the position or policy of the Department of Veterans Affairs or the United States government.
} 
opioids had increased fourfold between 2000 and 2014, and efforts are underway to limit their use. ${ }^{2}$ In 2017 , the DVA and the United States Department of Defense (DoD) published an updated guideline on opioid therapy for chronic pain that strongly recommends against initiation of longterm opioid use for chronic pain and recommends alternatives, including nonpharmacologic therapy. ${ }^{3}$

Nonpharmacologic therapy includes many integrative modalities, such as acupuncture, which has been shown to be effective for treating a variety of painful conditions. ${ }^{4}$ Battlefield Acupuncture (BFA), a subset of auricular acupuncture introduced by Niemtzow in $2001,{ }^{5}$ holds promise as a safe, inexpensive easily learned form of acupuncture and has been promoted by the DoD and the DVA for use in active military personnel and in veterans. ${ }^{6}$

While there is a relative paucity of published evidence to support BFA, ${ }^{7}$ more recently, evidence is accruing that this kind of acupuncture can be highly effective for pain reduction. ${ }^{8}$ However, little is also known about whether repeated applications are also similarly efficacious or if BFA in group visits produce results similar to individual sessions. In addition, primary care seems to be an ideal venue for administration for BFA, given that it is easily learned and patients with pain frequently see their primary care providers (PCPs). How to implement BFA within the context of a busy primary-care practice has not been well-described because challenges exist, such as lack of leadership support and maintaining access for other patients needing ongoing care. There are also the dictates of competing priorities: chronic disease management; health promotion; medication management; ordering tests and reviewing their results; ordering appropriate referrals; documentation; etc. BFA group clinics have been proposed as a potential solution (see Taylor et al., this issue, pp. 252-261). ${ }^{9}$

\section{MATERIALS AND METHODS}

\section{Training in Battlefield Acupuncture}

Primary care providers were offered 4 hours of BFA training as part of a 2-day "pain mini-residency" at the VA Connecticut Healthcare System, in West Haven, CT. A medical acupuncturist who was also trained in BFA led the BFA session. Providers undergoing this training could be credentialed in BFA if they had performed 5 procedures under supervision and then would be permitted to incorporate BFA into their clinical practices as desired.

\section{The Group Clinic}

After realizing the relative difficulty of incorporating BFA "as needed" within 1 of the author's (D.G.F.) primary care clinics, a BFA group clinic was created at the West Haven campus of the VA Connecticut Healthcare System.
Open to all patients within the VA Connecticut Healthcare System, the campus serves more than 45,000 veterans. Flyers and placards that provided the time and location of the group, diagrams of the ear, and information about how to enroll were placed within the medical center. Informational e-mails were sent to primary-care providers throughout the VA Connecticut, and advertisements were posted on the VA's electronic informational screens throughout the medical center. Patients were encouraged to contact the clinic directly and were also referred by other providers. The clinic was scheduled for 60 minutes and accommodated both previously scheduled patients and walk-ins; 30 minutes were allotted for documentation afterward. After the group clinic was started, some other providers within the medical center, who were credentialed to perform BFA, continued to perform BFA within the context of their nongroup clinics.

\section{Administration of BFA}

Group sessions began with a discussion of what BFA is, its risks and potential benefits, how it differs from traditional Chinese acupuncture, BFA's contraindications, and aftercare instructions. Patients were told that they were only eligible if they had had a previous evaluation of their pain by a medical practitioner; they were also told that BFA was only one modality in their entire care plans. After each eligible patient signed informed consent, gold Aiguilles semipermanentes (ASP) needles were sequentially inserted into the Cingulate Gyrus, Thalamus, Omega, Point Zero, and Shenmen points of both ears. When possible, background soothing music was played. At the conclusion of the group visit, patients were told that they could return to the weekly group visit whenever they wanted or needed to in the future. BFA was also done within the context of nongroup visits at the discretion of the BFA-trained provider for his or her own patients.

\section{Data Collection}

At each visit, the veteran's baseline pre-BFA selfreported pain (on a scale of 010) was captured, using the Defense and Veterans Pain Rating Scale (DVRPS) as well as the DoD/VA pain supplemental questions, ${ }^{10}$ asking how, in the past 24 hours, the pain has interfered with the patient's activity, sleep, and mood, and caused stress (also on a scale of 0-10). Immediate post-BFA self-reported pain levels, using the DVPRS, were also recorded. For individual visits, these measures were typically asked by the provider who administered the BFA procedure, while, for group sessions, the information was collected by someone other than the BFA provider, typically a volunteer. These data were recorded in the DVA electronic health record (EHR) system using a standard template incorporated into the "HealthFactors" function of the system. ${ }^{11}$ Information about the provider administering BFA and clinic location were available from the EHR. 
Existing data for patient characteristics (age, gender, and presence of an existing diagnosis of a chronic musculoskeletal condition associated with pain, using the International Statistical Classification of Diseases and Related Health Problems, 10th revised edition ${ }^{12}$ ) were in the VA electronic medical record.

\section{Data Analysis}

Changes in DVPRS pain scores before and immediately after BFA administration for each of the visits were assessed with $95 \%$ confidence intervals (CIs). To examine pain immediately before and immediately after BFA administration, a two-sided Wilcoxon signed-rank statistic was used to test if a change in pain scores was equal to 0 . An analysis of variance was used to compare change in pain, sleep, mood, stress, and activity across visit groups (first visit, visits $2-3$, visits 4 6 , and visits 7+) to identify potential group differences. Visits were included if any of the pain or supplemental measures were available. Visits with missing responses for an individual measure were excluded from the analysis of that measure. For all tests, 95\% CIs were produced. All analyses were conducted, using Stata 15.1 (College Station, TX).

\section{RESULTS}

Descriptive characteristics of the patients are shown in Table 1 . The patients were mostly men (92\%), and $71 \%$ had been previously diagnosed with a chronic musculoskeletal condition (Table 1). Over the study period, 284 unique veterans who received 753 BFA procedures were identified.

Table 1. Subjects' Characteristics

\begin{tabular}{lc}
\hline Characteristics & $\mathrm{N}(\%)$ \\
\hline Age & \\
$18-39$ & $25(9)$ \\
$40-49$ & $25(9)$ \\
$50-9$ & $48(17)$ \\
$60-69$ & $86(30)$ \\
$70-79$ & $78(27)$ \\
$80+$ & $22(8)$ \\
Gender & \\
Female & $23(8)$ \\
Male & $261(92)$ \\
History of chronic musculoskeletal condition & \\
None & $83(29)$ \\
Back & $115(40)$ \\
Fibromyalgia & $6(2)$ \\
Joint & $18(6)$ \\
Neck & $11(4)$ \\
Osteoarthritis & $31(11)$ \\
More than one condition & $20(7)$ \\
\hline
\end{tabular}

Table 2. Visit Patterns Among Patients and Providers

\begin{tabular}{lrrrrrr}
\hline & $Q 1$ & $Q 2$ & $Q 3$ & \multicolumn{2}{c}{ Q } & \multicolumn{2}{c}{ Q1 } \\
New providers \& visits & 2017 & 2017 & 2017 & 2017 & 2018 & Total \\
\hline New, unique providers & 6 & 5 & 3 & 4 & 9 & 27 \\
New, unique patients & 61 & 50 & 66 & 50 & 57 & 284 \\
Visits & 101 & 137 & 179 & 169 & 167 & 753 \\
\hline
\end{tabular}

Q, quarter.

Across the 6 items included in the 753 patient visits, there were 4 responses missing from the activity item, 4 from the sleep item, 3 from the mood item, 2 from the stress item, and 2 from the postpain assessment.

The volume of new patients attending the BFA clinic was steady over time with an average of 57 new patients per month being seen in the clinic (range: 50-66). The majority of the procedures, 567 (75\%), were administered or supervised by 1 provider, 1 of the study authors (D.G.F.). Overall, 27 providers administered at least 1 procedure with new providers being trained and administering at least 1 procedure during each quarter of the study period. The frequency of visits, new patients receiving an initial BFA procedure, and new providers administering BFA are shown by quarter over the study period in Table 2 .

Immediate pain reduction was common. Overall, of the 753 total patient encounters, a decrease in self-reported pain occurred in 616 encounters $(82.0 \%)$, there was no change in 73 encounters $(9.7 \%)$, and there was an increase in patients' pain' in 62 encounters $(8.3 \%)$. See Table 3, which shows results for the 751 encounters that had immediate postpain data. For 283 patients having their first BFA encounters, the results were similar: a decrease in pain score for 234 patients $(82.7 \%)$; no change in 27 patients $(9.5 \%)$; and an increase in pain in 22 patients $(7.8 \%)$. See Table 4.

Pain reduction was common whether the BFA was performed within the group setting or individually. Of the 552 group encounters, a decrease in pain occurred in 442 patients $(80.1 \%)$, there was no change in 64 patients $(11.6 \%)$, and there was an increase in pain in 46 patients $(8.3 \%)$. Similarly, in the nongroup setting, of the 199 encounters, a decrease in pain was noted in 174 patients $(87.4 \%)$, there was no change in 9 patients $(4.5 \%)$, and there was an increase in pain in 16 patients $(8.0 \%)$. See Table 5, which shows the visits for which there were immediate postpain data.

TAble 3. Response Rates for BFA Visits

\begin{tabular}{lcc}
\hline Results of BFA & \# of visits & \% of visits \\
\hline Decrease in pain & 616 & $82.0 \%$ \\
No change & 73 & $9.7 \%$ \\
Increase in pain & 62 & $8.3 \%$ \\
Total & $751^{\mathrm{a}}$ & - \\
\hline
\end{tabular}

${ }^{\mathrm{a}}$ Of the total 753 visits, only 751 had immediate post BFA-assessment data. BFA, Battlefield Acupuncture. 
Table 4. Response Rates for First BFA Visit Only

\begin{tabular}{lcc}
\hline & \# of people & \% of people \\
\hline Decrease in pain & 234 & $82.7 \%$ \\
No change & 27 & $9.5 \%$ \\
Increase in pain & 22 & $7.8 \%$ \\
Total & 283 & - \\
\hline
\end{tabular}

BFA, Battlefield Acupuncture.

$N=283$ as post pain level was missing for one patient's initial visit.

In the 178 patients undergoing their initial BFA in a group setting and in 105 undergoing their first BFA in individual sessions, reduction in pain occurred in 148 patients (83.1\%) versus 86 patients $(81.9 \%)$, there was no change in 19 patients $(10.7 \%)$ versus $8(7.6 \%)$, and there was an increase in pain in 11 patients $(6.2 \%)$ versus $11(10.5 \%)$ in the group versus individual settings, respectively (Tables $6 \mathrm{~A}$ and $6 \mathrm{~B}$ ).

Mean levels of the DVPRS pain assessments and supplemental questions are shown for each patient at the first visit and overall for all visits combined in Table 7A. In addition, pain assessments and supplemental measures are shown for visits $2-3$, visits $4-6$, and visits $7+$ higher in Table 7A. Overall, baseline pain scores for the 753 encounters averaged 6.9 (standard deviation [SD]: 2.3). For the 751 encounters with immediate post-BFA assessment data, patients experienced a-2.2-point (SD: 3.0) decrease in pain level. This change in pain level was consistent across first BFA visits ( -2.3 points; SD: 2.9$)$ with similar decreases in pain across visits $2-3,4-6$, and $7+$.

Similarly, pain assessments were examined grouped by baseline pain levels $\leq 6$ and 7-10 (Table 7B) and by whether BFA was performed as part of an individual clinic visit or a group visit (Table 7C). Absolute levels of pain reduction were greater in patients with the highest self-reported pain and did not seem to decrease in those who underwent repeated procedures.

\section{DISCUSSION}

Pain is a common patient complaint and efforts to ease suffering and minimize opioid use are of paramount impor-

Table 5. Total Response Rates for BFA in Group OR INDIVIDUAL SETTINGS

\begin{tabular}{lcccc}
\hline & \# of BFA & \% of BFA & \# of BFA & \# of BFA \\
& visits & visits & visits & visits \\
& for & for & for & for \\
Results of BFA & groups & groups & individuals & individuals \\
\hline Decrease in pain & 442 & $80.1 \%$ & 174 & $87.4 \%$ \\
No change & 64 & $11.6 \%$ & 9 & $4.5 \%$ \\
Increase in pain & 46 & $8.3 \%$ & 16 & $8.0 \%$ \\
Total & $552^{\mathrm{a}}$ & $100.0 \%$ & $199^{\mathrm{a}}$ & $100.0 \%$ \\
\hline
\end{tabular}

Of the total 753 visits, only 751 had immediate postpain data; hence the number of visits in this table add up to 751 .

BFA, Battlefield Acupuncture.
Table 6A. First BFA Visit Responses in Group Setting

\begin{tabular}{lcc}
\hline Result of BFA & \# of patients & \% of patients \\
\hline Decrease in pain & 148 & $83.1 \%$ \\
No change & 19 & $10.7 \%$ \\
Increase in pain & 11 & $6.2 \%$ \\
Total & 178 & $100 \%$ \\
\hline
\end{tabular}

BFA, Battlefield Acupuncture.

$N=283$ as post pain level was missing for one patient's initial visit.

TABle 6B. First BFA

Visit Responses in Nongroup Setting

\begin{tabular}{lcr}
\hline Result of BFA & \# of patients & \# of patients \\
\hline Decrease in pain & 86 & $81.9 \%$ \\
No change & 8 & $7.6 \%$ \\
Increase in pain & 11 & $10.5 \%$ \\
Total & 105 & $100.0 \%$ \\
\hline
\end{tabular}

BFA, Battlefield Acupuncture.

$N=283$ as post pain level was missing for one patient's initial visit.

tance. BFA has been promulgated as one of the integrative modalities that could help treat pain, and group visits have been touted as a way to provide the service efficiently to veterans. To the current authors' knowledge, this is the largest study published about BFA success and implementation. In addition, no prior study has compared the results of how successful BFA is when provided in group visits versus in individual settings.

With respect to efficacy, BFA was highly efficacious for reducing pain immediately. Group visits were an effective way to reduce self-reported pain immediately, including in patients with even the highest pain scores and highest selfreported impacts on function. In fact, patients with higher baseline self-reported pain had more decreases in pain than patients with lower baseline self-reported pain scores. Furthermore, for patients who desired continued BFA, it did not appear to have diminished efficacy with repeated applications.

BFA was not only effective in the group setting but was also effective when performed outside of the group, within the context of a one-on-one appointment. However, given the unadjusted, nonrandomized nature of the current observational study, the authors cannot draw conclusions that the group clinic results were inferior to the results of one-on-one visits, although further study would appear to be warranted. In addition, patients might have been more likely to report efficacy (because of a social desirability bias) when were required to respond verbally to an individual provider when BFA was performed individually, than when it was performed in the group, wherein written results were handed to a nonprovider.

Given that administering BFA in group clinics is a moreefficient use of patient and provider time than when BFA is administered within the context of one-on-one visits, the current authors believe that utilization of this type of clinic is a way to provide this service in a manner that minimizes a negative impact on access for other veterans who need medical services. 
Table 7A. Outcomes Associated with Timing of BFA Administration

\begin{tabular}{|c|c|c|c|c|c|}
\hline Results of BFA treatment & $\begin{array}{c}\text { First visit } \\
(\text { visits }=284) \\
(\text { patients }=284)\end{array}$ & $\begin{array}{c}\text { Visits } 2-3 \\
(\text { visits }=196) \\
(\text { patients }=121)\end{array}$ & $\begin{array}{c}\text { Visits 4-6 } \\
\text { (visits }=133 \text { ) } \\
\text { (patients }=56)\end{array}$ & $\begin{array}{c}\text { Visits } 7+ \\
(\text { visits }=140) \\
\text { (patients }=25)\end{array}$ & $\begin{array}{c}\text { Overall } \\
(\text { visits }=753) \\
(\text { patients }=284)\end{array}$ \\
\hline Pain level (pre) mean (SD) & $6.8(2.4)$ & $6.5(2.4)$ & $7.3(2.3)$ & $7.0(2.2)$ & $6.9(2.3)$ \\
\hline Pain level (post) mean (SD) & $4.5(2.7)$ & $4.5(2.6)$ & $4.9(2.6)$ & $5.1(2.0)$ & $4.7(2.5)$ \\
\hline Change mean (SD) & $-2.3(2.9)^{*}$ & $-2.1(3.2)^{*}$ & $-2.4(3.1)^{*}$ & $-2.0(2.8)^{*}$ & $-2.2(3.0)^{*}$ \\
\hline Interference with sleep (pre) mean (SD) & $5.6(3.0)$ & $5.5(3.0)$ & $6.1(3.0)$ & $7.0(2.8)$ & $5.9(3.0)$ \\
\hline Interference with activity (pre) mean (SD) & $6.3(2.8)$ & $6.1(2.7)$ & $6.7(2.8)$ & $6.8(2.5)$ & $6.4(2.7)$ \\
\hline Interference with mood (pre) mean (SD) & $5.7(2.9)$ & $5.5(2.9)$ & $6.2(3.0)$ & $6.9(2.4)$ & $6.0(2.9)$ \\
\hline Interference with stress (pre) mean (SD) & $5.9(2.9)$ & $5.6(2.9)$ & $6.3(2.8)$ & $6.8(2.4)$ & $6.0(2.8)$ \\
\hline
\end{tabular}

${ }^{*} P<0.001$; signed-rank test of change in pain scores not equal to 0 change.

BFA, Battlefield Acupuncture; SD, standard deviation.

Table 7B. Outcomes Associated with Baseline Pain Levels

\begin{tabular}{|c|c|c|}
\hline Results of BFA treatment & $\begin{array}{c}\text { Baseline pain level } \leq 6 \\
(\text { visits }=296)^{a} \\
(\text { patients }=114)\end{array}$ & $\begin{array}{c}\text { Baseline pain level 7-10 } \\
(\text { visits }=455)^{a} \\
(\text { patients }=169)\end{array}$ \\
\hline Pain level (pre) mean (SD) & $4.5(1.6)$ & $8.4(1.0)$ \\
\hline Pain level (post) mean (SD) & $3.7(2.5)$ & $5.3(2.4)$ \\
\hline Change mean (SD) & $-0.7(3.3)^{*}$ & $-3.2(2.3)^{*}$ \\
\hline Interference with sleep (pre) mean (SD) & $4.8(2.7)$ & $6.7(3.0)$ \\
\hline Interference with activity (pre) mean (SD) & $5.0(2.6)$ & $7.3(2.4)$ \\
\hline Interference with mood (pre) mean (SD) & $4.9(2.7)$ & $6.7(2.8)$ \\
\hline Interference with stress (pre) mean (SD) & $4.9(2.6)$ & $6.8(2.7)$ \\
\hline
\end{tabular}

${ }^{\mathrm{a}}$ Numbers of visits in this part of Table 7 show only those with immediate postpain data.

${ }^{*} P<0.001$; signed-rank test of change in pain scores not equal to 0 change.

BFA, Battlefield Acupuncture; SD, standard deviation.

Table 7C. Outcomes Associated with Group Location

\begin{tabular}{lcc}
\hline & $\begin{array}{c}\text { Administered in group clinic } \\
\text { (visits }=553) \\
\text { (patients }=178)\end{array}$ & $\begin{array}{c}\text { Administered during individual visit } \\
\text { (visits }=200) \\
(\text { patients }=106)\end{array}$ \\
\hline Pain level (pre) mean (SD) & $7.0(2.3)$ & $6.6(2.4)$ \\
Pain level (post) mean (SD) & $5.4(2.2)$ & $2.6(2.4)$ \\
Change mean (SD) & $-1.6(2.5) *$ & $-3.9(3.4)^{*}$ \\
Interference with sleep (pre) mean (SD) & $6.0(3.1)$ & $5.7(2.8)$ \\
Interference with activity (pre) mean (SD) & $6.7(2.6)$ & $5.7(2.9)$ \\
Interference with mood (pre) mean (SD) & $6.2(2.9)$ & $5.6(2.8)$ \\
Interference with stress (pre) mean (SD) & $6.2(2.8)$ & $5.6(2.8)$ \\
\hline
\end{tabular}

$* P<0.001$; signed-rank test of change in pain scores not equal to 0 change.

BFA, Battlefield Acupuncture; SD, standard deviation.

Limitations of the current study included its nonrandomized nature and the possibility of selection bias. Given that many patients were self-referred, the patients undergoing BFA in the study might have been predisposed to having a more favorable effect than had they presented with pain and been randomly assigned to either BFA or another treatment. According to the nature of the study design, the authors could not determine differences in BFA efficacy for acute or chronic pain, nor was it possible to provide data on the duration of efficacy for patients who derived immediate benefit.
Furthermore, the data were obtained by evaluation of standardized, templated, BFA progress notes, which might not have been used in every nongroup encounter. Using this method of data abstraction, free-texted data from progress notes could not be evaluated. However, there is no reason to believe that the results would be any different if BFA were to be documented outside of the templated progress notes. While this study was conducted at a large VA medical center, the authors do not know if results would be similar at other centers or in non-veterans. Finally, self-reported pain scores 
are highly variable among different patients, and functional improvements over time could be more meaningful.

However, the strengths of study include that, by comparing preintervention pain scores with immediate postintervention pain scores, the authors were likely to isolate the benefit of BFA only. Had pain scores been obtained at a later date, it would not have been possible to be sure whether improvements in pain were due to medical therapy, another intervention, or a natural healing process. In addition, it was possible to demonstrate that, for patients who come back for repeated BFA, the efficacy did not appear to diminish over time. Centers considering implementation of BFA in the future should plan for the fact that many patients come back for repeated procedures and centers should consider how these repeated procedures might affect patient access.

\section{CONCLUSIONS}

BFA can be highly efficacious for reducing pain for the overwhelming majority of patients exposed to this intervention, whether it is performed individually or in group clinics. Group clinics may be an effective and efficient way to deliver this important integrative modality. Whether or not this can lead to durability, an increased quality of life, and decreases in pharmacotherapy, especially opioid use, remains to be explored.

\section{ACKNOWLEDGMENTS}

This work was supported by the Department of Veterans Affairs Quality Enhancement Research Initiative program (PEC 16-354). The views expressed in this article are those of the authors and do not necessarily represent the position or policy of the Department of Veterans Affairs or the United States government.

All VHA authors of this manuscript attest that the activities that resulted in producing this article were not conducted as part of a research project, but as part of the nonresearch evaluation conducted under the authority of the VA Office of Patient Centered Care and Cultural Transformation (OPCC\&CT). The status of this work as quality improvement and not research was also confirmed following review by the Research and Development Committee at the VA Greater Los Angeles Healthcare System.

\section{AUTHOR DISCLOSURE STATEMENT}

No competing financial conflicts exist.

\section{REFERENCES}

1. U.S. House of Representatives. Between Peril and Promise: Facing the Dangers of VA's Skyrocketing Use of Prescription Painkillers to Treat Veterans. Washington, DC: House Committee on Veterans' Affairs, Subcommittee on Health; 2013.

2. Compton WM, Jones CM, Baldwin GT. Relationship between nonmedical prescription-opioid use and heroin use. $N$ Engl $J$ Med. 2016;374(2):154-163.

3. Rosenberg JM, Bilka BM, Wilson SM, Spevak C. Opioid therapy for chronic pain: Overview of the 2017 US Department of Veterans Affairs and US Department of Defense clinical practice guideline. Pain Med. 2018;19(5):928-941.

4. Vickers AJ, Cronin AM, Maschino AC, et al. Acupuncture for chronic pain: Individual patient data meta-analysis. Arch Intern Med. 2012;172(19):1444-1453.

5. Niemtzow RC. Battlefield Acupuncture. Med Acupunct. 2007; 19(4):225-228.

6. Walker PH, Pock A, Ling CG, Kwon KN, Vaughan M. Battlefield Acupuncture: Opening the door for acupuncture in Department of Defense/Veteran's Administration health care. Nurs Outlook. 2016;64(5):491-498.

7. Federman DG, Gunderson CG. Battlefield Acupuncture. Is it ready for widespread dissemination? South Med J. 2017;110(1): 55-57.

8. Federman DG, Poulin LM, Ruser CR, Kravetz JD. Implementation of shared medical appointments to offer battlefield acupuncture efficiently to veterans with pain. Acupunct Med. 2018;36(2):124-126.

9. Taylor SL, Giannitrapani K, Ackland PE, et al. Challenges and strategies for implementing battlefield acupuncture in the Veterans Administration: A qualitative study of provider perspectives. Med Acupunct. 2018;30(5):252-261.

10. Buckenmaier CC 3rd, Galloway KT, Polomano RC, McDuffie M, Kwon N, Gallagher RM. Preliminary validation of the Defense and Veterans Pain Rating Scale (DVPRS) in a military population. Pain Med. 2013;14(1):110-123.

11. Byrne CM, Mercincavage LM, Pan EC, Vincent AG, Johnston DS, Middleton B. The value from investments in health information technology at the U.S. Department of Veterans Affairs. Health Aff (Millwood). 2010;29(4):629-638.

12. World Health Organization (WHO). International Statistical Classification of Diseases and Related Health Problems, 10th rev ed. Geneva: WHO: 2015.

Address correspondence to: Daniel Glenn Federman, MD Department of Medicine VA Connecticut Healthcare System (11ACSL) 950 Campbell Avenue West Haven, CT 06516

E-mail: Daniel.federman@va.gov 